\title{
Comparison of Cortical Activation during Mahjong Game Play in a Video Game Setting and a Real-life Setting
}


Yanagisawa $^{7}$

${ }^{1}$ Graduate School of Medicine, Shinshu University, Matsumoto, Japan

2 Faculty of Education, Shinshu University, Nagano, Japan / Graduate School of Medicine, Shinshu Univesity,

Matsumoto, Japan

${ }^{3}$ School of Food and Nutritional Science, The University of Shizuoka, Shizuoka, Japan

${ }^{4}$ Kodomo Plus School of Support of Autism, Developmental disorder and ADHD, Tsukuba, Japan

${ }^{5}$ Shinshu University Faculty of Engineering, Nagano, Japan

${ }^{6}$ Center of General Education and Humanities, Tokyo University of Science, Japan

${ }^{7}$ Department of Infant Childcare, Matsumoto Junior College, Japan

*Corresponding author: Koji Terasawa, Graduate School of Medicine, Shinshu University, Matsumoto, Japan, Tel: +81-263-37-3376; E-mail: kterasa@shinshu-u.ac.jp Rec date: Jan 22, 2015; Acc date: Feb 26, 2015; Pub date: Feb 28, 2015

Copyright: (c) 2015 Fujimori S, et al. This is an open-access article distributed under the terms of the Creative Commons Attribution License, which permits unrestricted use, distribution, and reproduction in any medium, provided the original author and source are credited.

\begin{abstract}
The purpose of this study was to compare the hemodynamic changes that occur during Mahjong game play in virtual and real-life settings. Fourteen healthy right-handed men (average age \pm standard deviation; $36.7 \pm 14.9$ years) played: 1) a Mahjong solitaire game on a video console against virtual rivals; 2) a Mahjong game against human opponents without conversation; and 3) a Mahjong game against human opponents with conversation. We measured oxygenated hemoglobin concentration at 44 locations over both hemispheres during Mahjong game play in each setting using near-infrared spectroscopy. The increase in oxygenated hemoglobin concentration at several locations, including Broca's area, the somatosensory cortex, the somatosensory association cortex, the supramarginal gyrus part of Wernicke's area, the primary and auditory association cortex, the angular gyrus part of Wernicke's area, and the associative visual cortex was greater during game play in the real-life settings than during game play in the video game setting. There were no significant differences during game play in real-life settings without and with conversation. Each cortical area correlated with broad or specific areas. The common correlation areas were found at Angular gyrus part of Wernicke's area of left hemisphere during real-life settings without and with conversation, but not during game play in a video game setting. These results suggest that the brain responds differently to game play in real world and virtual world settings, and indicate that comparison of games played in the virtual world and the real world may be an effective model to enhance understanding of the effects of video game on the brain.
\end{abstract}

Keywords: Near-infrared spectroscopy; Videogames; Oxygenated hemoglobin concentration; Angular gyrus; Supramarginal gyrus

\section{Introduction}

Several studies have used game-playing tasks to study cerebral functions and, recently, there has been much interest on the effects of playing video games on brain activity. At present, the effects of playing video games on the brain activity are not clearly understood. Although many adverse effects of playing video games have been reported $[1,2]$, videogames may also have beneficial effects on the brain such as mental treatment, visual training [3,4]. There are various types of video games, and it may be expected that they have different effects on brain activity. In addition, their effect on the brain may differ according to the age of the participant [5]. Little is known about whether the brain responds differently to a video game as it does to a live version of the same game. However, this comparison of the games played in the virtual world and in the real world may be an effective model to enhance understanding of the effects of video game on brain activity. Near-infrared spectroscopy (NIRS) is a functional brain imaging technique in which cerebral blood volume changes are monitored through oxygenated hemoglobin (oxy-Hb), deoxygenated hemoglobin (deoxy-Hb), and total hemoglobin (total- $\mathrm{Hb}$ ) concentrations. The basic principle of NIRS lies in the different wavelength of near infrared light being translated to changes in oxy$\mathrm{Hb}$ and deoxy- $\mathrm{Hb}$ concentration. NIRS is noninvasive, and can be conducted in a natural setting, with the subject in a sitting position, with eyes open and speaking. As such, NIRS is well suited to functional neuroimaging studies. Brain activity involves regional changes in blood flow and oxygenation, and can be inferred from NIRS data based on the assumption that an increase in blood flow results in an increase in local oxygenation. Studies using NIRS have shown increased oxy- $\mathrm{Hb}$ in the forebrain during cognitive activation [6-10].

NIRS therefore allows the measurement of distinct regional cerebral responses and typical hemodynamic responses during functional tasks $[5,11,12]$.

The sensorimotor areas, particularly the motor areas, that are activated when an infant performs an action in real are activated too, on the other hand, an action observation in virtual such as video seeing, are not activated [13]. This suggests that the infants' brain responds another way whether real or virtual. However, this does not 
appear to be the case in adults. There is evidence that brain activity during video game playing is different in adults and children. During video game playing that involved frequent tapping, bilateral prefrontal total-Hb concentration increased in adults but decreased in children [14], and the hemodynamic changes between the prefrontal and the bilateral motor cortex were correlated in adults but were not children [15]. In addition, it has been reported that there is a sustained decrease in oxy-Hb concentration in the dorsal prefrontal cortex during video game playing in children. However, playing video games that involve frequent tapping increases not only blood pressure, but heart rate and oxy-Hb concentration, and increases blood flow in the motor cortex area. It has been also reported that the attention to visual stimuli is negatively associated with the $\mathrm{Hb}$ signal in the dorsal prefrontal cortex [16].

Mahjong is a Chinese game that is similar to a card game. Four players sit around a table and play using tiles that are carved with Chinese marks on one side. Mahjong provides a suitable paradigm for studying the differences in cerebral activation patterns in different game playing situations. Thus, we hypothesized that the first task; Mahjong video game is needless frequent tapping, would lead to the cerebral response of video game excluding (without) the reflection of tapping action. Second and third task; playing Mahjong game with four people face to face without and with conversation, respectively would show the cerebral response of the game itself and the influences of conversation. Virtual environments have become common in our society, so the difference with real life may not be clear. However, there are few studies that pay attention to the quantity of brain blood flow of a virtual act during real action. Therefore this study pays attention to the oxy- $\mathrm{Hb}$ of the brain and clarifies the difference between a real mahjong and a virtual video game.

The purpose of the present study was to examine the hemodynamic changes that occur when playing Mahjong in different settings. These results will enable us to compare brain responses that occur in the real and the virtual world, and will thus enhance our understanding of the effects of video games on the brain.

\section{Methods}

\section{Subjects}

Fourteen healthy right-handed men (average age \pm SE; $36.7 \pm 14.9$ years, range $20-67$ years) who had no patient's history were recruited as volunteer subjects. All subjects were familiar with both the traditional Mahjong game and the video Mahjong game. Prior to participating in the experiment, all subjects gave their written informed consent. This study was approved by the Ethics Committee of School of Medicine, Shinshu University, Japan.

\section{Study protocol}

Each subject played the Mahjong game in three settings: (1) solitaire video game (Tasuke SPIRITS II, Nintendo DS, Nintendo, Kyoto, Japan) play against the three virtual opponents on a computer screen (VG); (2) real-life play against three opponents around a table without conversation (TGN); and (3) real-life play against three opponents players around a table with conversation (TGC). The order of playing was randomized, and the counterbalanced among the subjects on the same day. In each setting, the game was played for $16 \mathrm{~min}$, consisting of four rest periods and three play periods in the following sequence: $10 \mathrm{~s}$ rest, $300 \mathrm{~s}$ play, $20 \mathrm{~s}$ rest, $300 \mathrm{~s}$ play, $20 \mathrm{~s}$ rest, $300 \mathrm{~s}$ play, $10 \mathrm{~s}$ rest. To avoid the confounding effect of head tilt, subjects were instructed to look straight ahead at a wall without looking up or down during rest periods.

\section{NIRS acquisition}

NIRS was performed throughout Mahjong game play and rest periods using a multi-channel near-infrared spectroscope (OMM-3000, Shimadzu, Kyoto, Japan). Subjects wore a head cap that covered the whole head (Figure 1). The head cap contained 15 optodes (corresponding to 22 channels) over both hemisphere, by the international 10/20 system. Left hemisphere has 8 emitter optodes (as red points) and has 7 detector optodes (as white points), right also has the optodes as the same way, symmetrically. Optodes areset at intervals of $3 \mathrm{~cm}$ on the head cap which shown in Fig1. The each channel's rate of sampling is about $8 \mathrm{~Hz}$.

Oxy-Hb concentration was observed as the indicate of hemodynamic change which investigated to be sensitive $[17,18]$. This change is researched by three wavelength of near-infrared equipment which called NIRS. As Takeuchi reported [19], and equipment manufacturer announced that the NIRS's irradiation power was less $1 \mathrm{~mW}$. The hemodynamic change from control base- line were estimated based on a modified Lambert-Beer law [20]. The depth of light stream to inside the inner head of the brain from the scalp about $1.5 \pm 0.5 \mathrm{~cm}[21]$.

\section{NIRS analysis}

The average changes in the $\mathrm{Hb}$ concentration in each channel were calculated for the pre-task baseline (=the first rest) of $10 \mathrm{~s}$ and task segments of 3 times 300s for each subject's 3 types of tasks as T-score by Student' s t-test. The data were analyzed by analysis of variance (ANOVA). After ANOVA, Bonferroni/Dunn test were done as post hoc test. Correction for the false discovery rate (FDR) was performed, since this in commonly done in NIRS data analysis.

The NIRS data analyzed with a least-squares estimation by a general linear model $[22,23]$. Takamoto reported [24] that the time course of oxy-Hb in each game setting was correlated boxcar function's design matrix. Gaussian kernel convoluted the equation, data and the matrix [25]. The periods of game play were contrasted against the rest periods for each game setting (VG, TGN and TGC) using a one-tailed $t$ test. oxy-Hb in each channel during rest and game play periods in each trial was calculated using a one-tailed $t$-test.

\section{Statistical analysis}

The resulting $t$ values were compared across game setting (VG, TGN, TGC) using a one-way analysis of variance. Post hoc tests (pairwise comparisons) were made using Bonferroni correction. The correlation among NIRS channels with significant activation for all three tasks was evaluated by Pearson's correlation coefficients. To examine the differences in inter-channel correlations among the three game settings, we classified channels that had common correlations (with $\mathrm{P}<0.01)$ into one of four categories: $(1)$ in all three game settings; (2) in VG and TGN; (3) in VG and TGC; and (4) in TGN and TGC. Criterion for significance was set at $\mathrm{P}<0.05$ for all tests. 
Citation: Fujimori S, Terasawa K, Murata Y, Ogawa K, Tabuchi H, et al. (2015) Comparison of Cortical Activation during Mahjong Game Play in a Video Game Setting and a Real-life Setting. Biochem Anal Biochem 4: 164. doi:10.4172/2161-1009.1000164

A

B

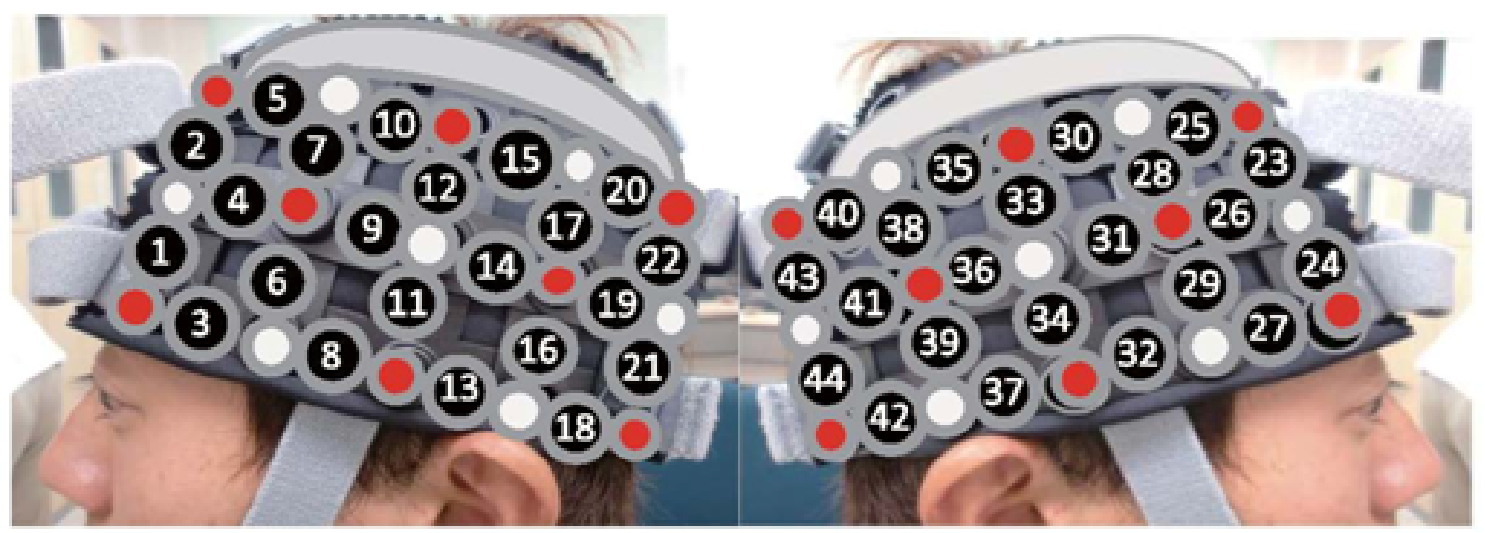

Figure 1: Location of near-infrared spectroscopy probes on the scalp. Near-infrared spectroscopy data were obtained using a 44-channel spectrometer. Subjects wore a head cap such that channels 1-22 were placed over the left hemisphere (A) and channels. 23-44 were placed over the right hemisphere (B).

\section{Results}

In each of the 14 subjects, we measured oxy hemodynamics in the cerebral cortex by NIRS during the three types of tasks (VG, TGN, TGC). Measurement points were 22 channels in the left hemisphere and 22 channels in the right hemisphere (total; 44). $t$-Values calculated by measurement data showed that oxy-Hb of 13 of the subjects decreased more during VG than during TG.

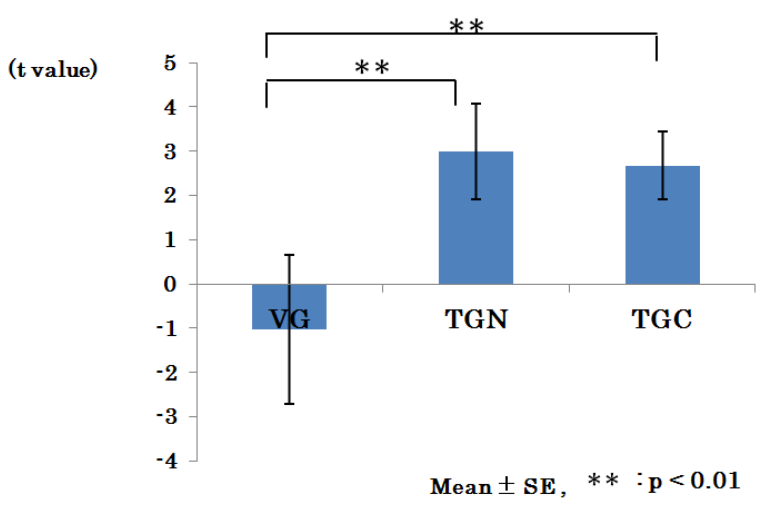

Figure 2: Average of $t$-value of oxygenated hemoglobin concentration under the three tasks. The task playing mahjong by video game (VG), the task playing traditional mahjong game without conversation (TGN), the task playing traditional mahjong game with conversation (TGC).

Figure 2 shows that the average $\mathrm{t}$-values overall for each type of task were: VG; -1.03, TGN; 2.99 and TGC; 2.67 , and ANOVA showed significant differences $(\mathrm{p}<0.001)$. VG was significant lower than during TGN $(\mathrm{p}<0.01)$ or TGC $(\mathrm{p}<0.01)$.

oxy-Hb in each channel during each game setting is shown in Table 1 . There was a main effect of game setting for $\Delta$ oxy- $\mathrm{Hb}$ in channels 6 $(\mathrm{P}<0.05), 14(\mathrm{P}<0.05), 16(\mathrm{P}<0.001), 18(\mathrm{P}<0.01), 19(\mathrm{P}<0.001), 21$ $(\mathrm{P}<0.01)$, and $22(\mathrm{P}<0.05)$ in the left hemisphere, and channels 36 $(\mathrm{P}<0.05), 38(\mathrm{P}<0.05), 39(\mathrm{P}<0.001), 41 \quad(\mathrm{P}<0.001), 42(\mathrm{P}<0.01), 43$ $(\mathrm{P}<0.05)$, and $44(\mathrm{P}<0.001)$ in the right hemisphere (Table 1) (Figure $3)$.
A

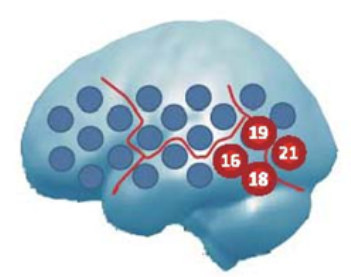

B

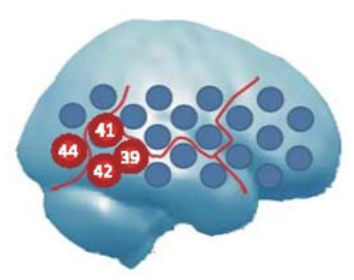

Figure 3: Schematic indicating the channels in which there was a main effect of setting on the change in oxy-Hb concentration during Mahjong game. (A) Left hemisphere. (B) Right hemisphere.

The areas of distinctions among the 3 tasks as the results of one-way ANOVA. Broca's area (ch6), Somatosensory Cortex (ch14, 36), Somatosensory association cortex (ch38), Supramarginal gyrus part of Wernicke's area (ch16, 39), Primary and Auditory Association Cortex (ch18, 42), Angular gyrus part of Wernicke's area (ch19, 21, 41, 44), Associative visual cortex (ch22, 43), respectively. 
Citation: Fujimori S, Terasawa K, Murata Y, Ogawa K, Tabuchi H, et al. (2015) Comparison of Cortical Activation during Mahjong Game Play in a Video Game Setting and a Real-life Setting. Biochem Anal Biochem 4: 164. doi:10.4172/2161-1009.1000164

Page 4 of 8

These channels were located over Broca's area (channel 6), the somatosensory cortex (channels 14 and 36), the somatosensory association cortex (channel 38), the supramarginal gyrus part of Wernicke's area (channels 16 and 39), the primary and auditory association cortex (channels 18 and 42), the angular gyrus part of Wernicke's area (channels 19,21, 41 and 44), and the associative visual cortex (channels 22 and 43). Post hoc tests revealed that oxy-Hb during VG was lower than during TGN or TGC (Table 1). There were no significant differences between oxy-Hb during TGN and TGC in any channel.

The classification of channels with common correlations is shown in Table 2. Broca's area (channel 6, BA44, 45, 46) was correlated with channels 3, 9, and 29 in all game settings. Among the association channels, ch6 and 29 were the same area in both hemisphere. The association of both hemispheres was observed in the somatosensory cortex (channels 14 and 36, BA1, 2 and 3), the somatosensory association cortex (channel 38, BA7), and the supramarginal gyrus part of Wernicke's area (channels 16 and 39, BA40) in VG and TGN tasks, and in the associative visual cortex (channels 22 and 43) in TGN and TGC tasks.

The primary and auditory association cortex (channels 18 and 42 , BA41 and 42) was correlated with in VG and TGN tasks. In the angular gyrus part of Wernicke's area (channels 19, 21, 41 and 44, BA39), the common correlations in the TGN and TGC tasks were in the left hemisphere (channels 19 and 21), but not in the right hemisphere (channels 41 and 44).

\begin{tabular}{|c|c|c|c|c|c|c|c|c|c|c|c|c|c|}
\hline \multicolumn{14}{|c|}{ Changes in oxy-Hb concentration during 3 tasks; VG, TGN and TGC } \\
\hline \multirow[b]{3}{*}{ Left } & \multirow{3}{*}{$\begin{array}{l}\text { Channel } \\
\text { ch1 }\end{array}$} & \multirow{2}{*}{\multicolumn{3}{|c|}{$\begin{array}{l}\text { VG } \\
\text { Mean } \pm S E\end{array}$}} & \multirow{2}{*}{\multicolumn{3}{|c|}{$\begin{array}{l}\text { TGN } \\
\text { Mean } \pm \text { SE }\end{array}$}} & \multirow{2}{*}{\multicolumn{3}{|c|}{$\begin{array}{l}\text { TGC } \\
\text { Mean } \pm S E\end{array}$}} & \multirow{3}{*}{$\begin{array}{l}\text { ANOVA } \\
\text { P value } \\
0.614\end{array}$} & \multirow{3}{*}{$\begin{array}{l}\text { post hoc test } \\
\text { VG vs. TGN }\end{array}$} & \multirow{3}{*}{ VG vs. TGC } \\
\hline & & & & & & & & & & & & & \\
\hline & & 8.59 & \pm & 3.98 & 5.13 & \pm & 1.5 & 5.76 & \pm & 1.6 & & & \\
\hline & $\operatorname{ch} 2$ & -2.48 & \pm & 3.2 & 2.99 & \pm & 1.6 & 0.8 & \pm & 2.2 & 0.292 & & \\
\hline & $\operatorname{ch} 3$ & 1.13 & \pm & 3.33 & 4.87 & \pm & 1.5 & 5.76 & \pm & 1.6 & 0.329 & & \\
\hline & ch4 & 4 & \pm & 3.16 & 5.05 & \pm & 1.4 & 3.76 & \pm & 2.3 & 0.92 & & \\
\hline & ch5 & 2.2 & \pm & 3.61 & 0.76 & \pm & 1.4 & -0.55 & \pm & 1.7 & 0.729 & & \\
\hline & ch6 & -2.68 & \pm & 2.12 & 3.39 & \pm & 1.9 & 4.03 & \pm & 1.7 & 0.030 * & & $\ddagger$ \\
\hline & $\operatorname{ch} 7$ & 3.1 & \pm & 3.15 & 4.07 & \pm & 1.6 & 1.61 & \pm & 1.7 & 0.742 & & \\
\hline & ch8 & 3.26 & \pm & 3.38 & 4.3 & \pm & 1.7 & 2.69 & \pm & 1.6 & 0.888 & & \\
\hline & $\operatorname{ch} 9$ & 0.69 & \pm & 2.31 & 5.46 & \pm & 1.8 & 6.72 & \pm & 2.1 & 0.106 & & \\
\hline & $\operatorname{ch} 10$ & 3 & \pm & 3.19 & 2.85 & \pm & 1.6 & 1.13 & \pm & 1.5 & 0.806 & & \\
\hline & ch11 & -0.63 & \pm & 3.28 & 4.93 & \pm & 1.6 & 1.89 & \pm & 1.2 & 0.217 & & \\
\hline & $\operatorname{ch} 12$ & 1.61 & \pm & 2.66 & 5.54 & \pm & 1.9 & 4.08 & \pm & 1.5 & 0.405 & & \\
\hline & $\operatorname{ch} 13$ & 0.6 & \pm & 3.04 & 4.9 & \pm & 1.5 & 2.67 & \pm & 1.3 & 0.361 & & \\
\hline & $\operatorname{ch} 14$ & -1.25 & \pm & 2.48 & 6.27 & \pm & 1.8 & 6.86 & \pm & 1.9 & 0.014 * & $\dagger$ & $\ddagger$ \\
\hline & ch15 & 2.99 & \pm & 3.68 & 4.97 & \pm & 1 & 1.65 & \pm & 1.5 & 0.614 & & \\
\hline & $\operatorname{ch} 16$ & -5.8 & \pm & 2 & 4.49 & \pm & 1.9 & 5.55 & \pm & 1.5 & $0.000^{* *}$ & †† & $\ddagger \ddagger$ \\
\hline & ch17 & -1.68 & \pm & 4.27 & 4.88 & \pm & 1.6 & 4.97 & \pm & 1.5 & 0.165 & & \\
\hline & $\operatorname{ch} 18$ & -3.8 & \pm & 1.96 & 3.54 & \pm & 1.9 & 5.51 & \pm & 1 & $0.001^{\text {** }}$ & †† & 㧊 \\
\hline & $\operatorname{ch} 19$ & -9.99 & \pm & 1.95 & 2.17 & \pm & 2.1 & 4.38 & \pm & 1.3 & $0.000^{* *}$ & †† & 抽 \\
\hline & $\operatorname{ch} 20$ & -0.6 & \pm & 3.23 & 1.89 & \pm & 1.8 & 1.48 & \pm & 1.5 & 0.719 & & \\
\hline & ch21 & -6.67 & \pm & 2.2 & 1.81 & \pm & 2.2 & 4.17 & \pm & 1.5 & $0.001^{\text {** }}$ & $\dagger$ & 㧊 \\
\hline & ch22 & -7.23 & \pm & 2.85 & -0.3 & \pm & 2.1 & 1.28 & \pm & 1.2 & 0.020 * & & $\ddagger$ \\
\hline Right & $\operatorname{ch} 23$ & -1.47 & \pm & 3.12 & 1.32 & \pm & 1.4 & -2.55 & \pm & 1.5 & 0.426 & & \\
\hline & ch24 & 4.41 & \pm & 2.34 & 4.04 & \pm & 1.5 & 0.72 & \pm & 1.6 & 0.308 & & \\
\hline & $\operatorname{ch} 25$ & -2.2 & \pm & 3.11 & 0.09 & \pm & 1.2 & -2.67 & \pm & 1.3 & 0.62 & & \\
\hline & $\operatorname{ch} 26$ & -1.53 & \pm & 2.51 & 2.69 & \pm & 1.4 & -0.86 & \pm & 1. 36 & 0.23 & & \\
\hline
\end{tabular}


Citation: Fujimori S, Terasawa K, Murata Y, Ogawa K, Tabuchi H, et al. (2015) Comparison of Cortical Activation during Mahjong Game Play in a Video Game Setting and a Real-life Setting. Biochem Anal Biochem 4: 164. doi:10.4172/2161-1009.1000164

Page 5 of 8

\begin{tabular}{|c|c|c|c|c|c|c|c|c|c|c|c|c|}
\hline ch27 & 4.95 & \pm & 3.37 & 3.12 & \pm & 1.8 & 4.61 & \pm & 1.2 & 0.84 & & \\
\hline $\operatorname{ch} 28$ & -0.11 & \pm & 1.74 & 3.41 & \pm & 1.2 & 2.31 & \pm & 1. 22 & 0.208 & & \\
\hline $\operatorname{ch} 29$ & 0.03 & \pm & 2.4 & 4.05 & \pm & 2 & 4.41 & \pm & 1.8 & 0.262 & & \\
\hline $\operatorname{ch} 30$ & 2.73 & \pm & 2.63 & 2.58 & \pm & 1.5 & 0.85 & \pm & 1 & 0.724 & & \\
\hline ch31 & -0.56 & \pm & 2.26 & 2.93 & \pm & 1.9 & 2.88 & \pm & 1.1 & 0.31 & & \\
\hline ch32 & 1.99 & \pm & 2.55 & 3.16 & \pm & 1.9 & 3.32 & \pm & 1.2 & 0.873 & & \\
\hline $\operatorname{ch} 33$ & 0.6 & \pm & 2.35 & 4 & \pm & 1.8 & 2.36 & \pm & 1.1 & 0.422 & & \\
\hline $\operatorname{ch} 34$ & -0.42 & \pm & 2.37 & 3.01 & \pm & 1.8 & 3.28 & \pm & 1.1 & 0.29 & & \\
\hline ch35 & 2.9 & \pm & 3.24 & 4.86 & \pm & 1.5 & 0.7 & \pm & 1.8 & 0.45 & & \\
\hline $\operatorname{ch} 36$ & -1.4 & \pm & 1.82 & 3.76 & \pm & 1.5 & 4.87 & \pm & 1.1 & $0.012^{*}$ & & $\ddagger$ \\
\hline $\operatorname{ch} 37$ & 0.19 & \pm & 2.44 & 2.29 & \pm & 1.7 & 3.06 & \pm & 1.5 & 0.555 & & \\
\hline ch38 & -3.44 & \pm & 2.26 & 2.78 & \pm & 1 & 1.24 & \pm & 1.5 & 0.031 * & $\dagger$ & \\
\hline ch39 & -5.57 & \pm & 1.66 & 2.41 & \pm & 1.7 & 3.56 & \pm & 1 & $0.000^{* *}$ & †† & 拉 \\
\hline ch40 & -3.14 & \pm & 3.6 & -0.87 & \pm & 1.1 & 1.37 & \pm & 1.2 & 0.383 & & \\
\hline ch41 & -8.61 & \pm & 1.98 & 1.04 & \pm & 1.7 & 4 & \pm & 1.1 & $0.000^{* *}$ & †† & 㧊 \\
\hline ch42 & -6.43 & \pm & 1.61 & 1.13 & \pm & 1.9 & 2.15 & \pm & 1.1 & $0.001^{* *}$ & t† &  \\
\hline ch43 & -7.56 & \pm & 3.26 & -2.97 & \pm & 1.7 & 0.51 & \pm & 1.1 & $0.045^{*}$ & & $\ddagger$ \\
\hline ch44 & -9.26 & \pm & 1.79 & -1.27 & \pm & 1.5 & 1.32 & \pm & 1.2 & $0.000^{* *}$ & †† & 㧊 \\
\hline
\end{tabular}

Table 1: The change in oxy-Hb concentration during Mahjong game play in a video game setting (VG), and a real-life setting without conversation (TGN) and with conversation (TGC). Data indicate mean $\pm \mathrm{SE}$. $\mathrm{P}$ values indicate the main effect of game play setting. ${ }^{\dagger} \mathrm{P}<0.05$ and ${ }^{\dagger \dagger} \mathrm{P}<0.01$ for post hoc test of $\mathrm{VG}$ vs. TGN. ${ }^{\ddagger} \mathrm{P}<0.05$ and 㧊 $\mathrm{P}<0.01$ for post hoc test of VG vs. TGC. There were no significant post hoc differences between TGN and TGC.

\section{Discussion}

The major finding of this study was that the increase in oxy-Hb in Broca's area, the somatosensory cortex, the somatosensory association cortex, the supramarginal gyrus part of Wernicke's area, the primary and auditory association cortex, the angular gyrus part of Wernicke's area, and the associative visual cortex was lower during Mahjong game play in a video game setting than during Mahjong game play in a reallife setting. These results suggest that the video game setting is related to lower activation of the cortical areas associated with language, somatosensory, and visual processing and lower activation of a bilateral network of regions in the temporal and parietal lobes, than real-life game play settings. According to the Broadman map, it has well known that Broca's area is the area for the cognitive control of memory, and dissociable controlled retrieval and generalized selection, that Somatosensory area is the receiving area of touch, that Somatosensory association cortex is the receiving area of lower limb movement, that Supramarginal gyrus part of Wernicke's area is the control area of language and cognition, that Angular gyrus, part of Wernicke's area is memory and space cognition, that Auditory area is the control area of sound, and that Visual area is the auditory area. Taken together, real setting (e.g. TGN and TGC) was used visual, auditory, and touch information more than videogame setting (VG), suggesting that cognition of sense such as visual, auditory, and touch was controlled in real-life setting more than video game setting. The real-life setting probably needs to exchange the interactive information from visual, auditory, and touch sense, compared to the video game setting. Since the real-life setting need to prospect the partners' action, recognize self situation, and control self action, the video game setting seems to lack of these process.

The results of the present study allow us to compare the cortical responses between the virtual and the live world [13]. Cortical activity was similar during Mahjong game play in a real-life setting with and without conversation. This is contrast to a previous study that reported that realistic face-to-face conversation was accompanied by robust activation, represented by increases in oxy- $\mathrm{Hb}$ concentration, of the frontal and superior temporal regions [26]. Our results did show that oxy-Hb concentration during game play with conversation tended to be higher than during game play without conversation, however, this was not significant on post hoc tests. Probably even if there is not a conversation, the meeting with others influences it, and the activity of the Broca's area is the same as with conversation.

To examine the distinction of reflex relations among the three tasks, we performed the correlation analysis. In case that the results of positive correlations in VG interpreted the association of lower levels in oxy- $\mathrm{Hb}$ concentration, because the oxy- $\mathrm{Hb}$ concentration during VG was decreased in those channels. In contrast, positive correlation of TGN and TGC indicated the increased activation. Even so, common correlation areas in VG and TGN or in TGN and TGC were Broca's area, the somatosensory cortex, the somatosensory association cortex, and the supramarginal gyrus part of Wernicke's area. Although it is possible that each channel is reflected or influenced by two or three neighboring channels [27], our results showed that each area of 
Citation: Fujimori S, Terasawa K, Murata Y, Ogawa K, Tabuchi H, et al. (2015) Comparison of Cortical Activation during Mahjong Game Play in a Video Game Setting and a Real-life Setting. Biochem Anal Biochem 4: 164. doi:10.4172/2161-1009.1000164

Page 6 of 8

channel might be reflected or crosstalk broad or specific brain areas. It is possible that Broca's area, the somatosensory cortex, the somatosensory association cortex, and the supramarginal gyrus part of
Wernicke's area stimulated or reflected the activity of other brain areas, including areas in the opposite hemisphere, during game play.

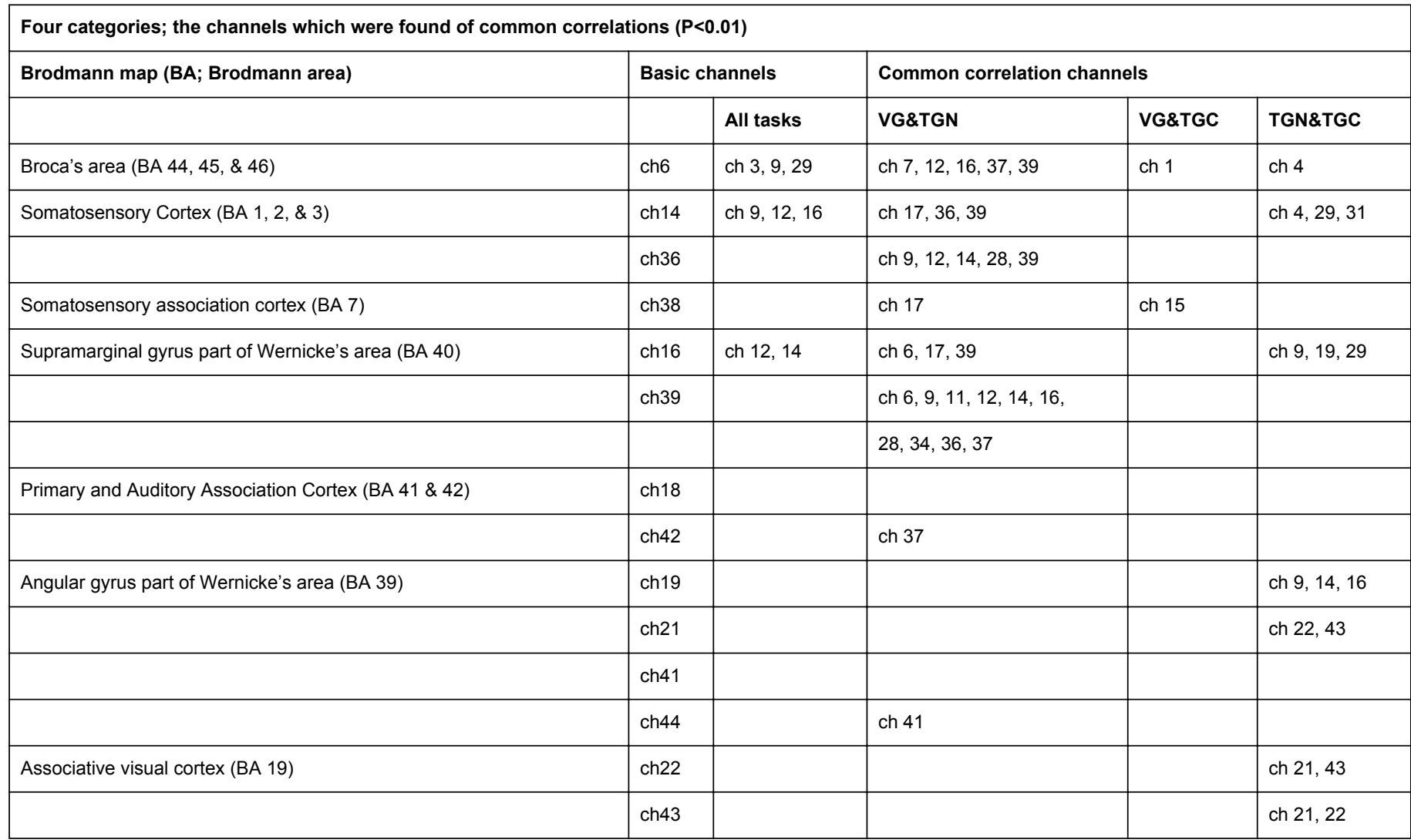

Table 2: Channels that had common correlations $(\mathrm{P}<0.01)$ in all Mahjong game play settings (VG, TGN and TGC), in VG and TGN settings, in VG and TGC settings, and in TGN and TGC settings. VG: video game setting; TGN: real-life setting without conversation; TGC: real-life setting with conversation; BA: Brodmann area. Red numbers denote the same areas of the contralateral hemisphere on the basic channels.

Interestingly, at the angular gyrus part of Wernicke's area in the left hemisphere, there were common correlation areas in TGN and TGC but not in VG. This might indicate that the angular gyrus part of Wernicke's area in the left hemisphere is activated and connected to other brain areas only during face-to-face game playing game settings, and not in virtual game playing settings. This was the major distinction between real play game and virtual play game in the current results. Geschwind discussed that the angular gyrus is a visual memory center for words, and by seeing and hearing, written word memory that is formed and stocked [28]. The activity of the angular gyrus is also frequently modulated during number processing [29], and involved in processes working memory [30] and cognition, as the results of linguistic and mathematical competence [31]. Passive viewing and perception of familiar symbols increased activation in the left angular gyrus to a greater extent than passing viewing of unfamiliar symbols did [32], and the left angular gyrus and superior temporal gyri activated more to the symbolic than the nonsymbolic [33]. In addition, ADHD subjects were found significance reduced volume of the right angular gyrus [34], Alzheimer's disease patients showed significant glucose hypometabolism in angular gyrus [35] and multiplayer online role-playing games showed a significantly decreased activations in the angular gyrus [36]. These findings suggest that the left angular gyrus subserves a more fundamental cognitive function in mathematical thinking than just arithmetic fact retrieval [31], and our results could therefore indicate that the cognition of numbers and/or letters was activated during TGN and TGC, but not VG.

In contrast, playing or watching the traditional Chinese gambling game Mah-Jong triggered epileptic seizures [37]. Epileptic seizures are known to be induced in some patients by flickering lights and color patterns, reading, language, movement, thinking, decision making, tapping and touching, and related stress [38]. Extensive observations show the frequent occurrence of provoked seizures in malformations of cortical development and in recently defined conditions such as familial or sporadic lateral temporal epilepsy [39]. However the mechanism that causes reflex epilepsy is not clear. We would like to further study epileptic seizures including the effects made by playing mahjong.

\section{Conclusion}

Our NIRS study demonstrated that the increase in oxy-Hb concentration in Broca's area, the somatosensory cortex, the somatosensory association cortex, the supramarginal gyrus part of Wernicke's area, the primary and auditory association cortex, the 
angular gyrus part of Wernicke's area, and the associative visual cortex was lower during Mahjong game play in a video game setting than during Mahjong game play in a real-life setting. Our results also indicate the feasibly of NIRS in determining temporal relations among brain areas in opposite hemispheres. Further investigation of relations and other brain areas involving left angular gyrus will elucidate the neuronal processes and distinctions between real and virtual world. In future study, we pay attention about the influence of age whether there is any difference in behavior among young and old players gyrus [35] and multiplayer online role-playing games showed a significantly decreased activations in the angular gyrus [36]. These findings suggest that the left angular gyrus subserves a more fundamental cognitive function in mathematical thinking than just arithmetic fact retrieval [31], and our results could therefore indicate that the cognition of numbers and/or letters was activated during TGN and TGC, but not VG.

\section{Acknowledgements}

The authors would like to thank Yasuhito Yoshizawa, Koki Nakajima, Kenichi Nemoto, Tomio Matsumura, Hiroshi Miura and Masao Okuhara for technical support. Kishiko Ogawa and Koji Terasawa was supported by a Grant-in-Aid for the Scientist (Houga: 23650426, KibanA: 25257101) of the Ministry of Education, Culture, Sports, Science and Technology of Japan. This study was supported grant of the Preventive Medical Center of Shinshu University Hospital from Ministry of Education, Culture, Science and Technology.

\section{References}

1. Cleary AG, McKendrick H, Sills JA (2002) Hand-arm vibration syndrome may be associated with prolonged use of vibrating computer games. BMJ 324: 301.

2. Griffiths MD (2002) Playing video games seems to have few serious acute adverse effects on health. BMJ 324: 1159.

3. Achtman RL, Green CS, Bavelier D (2008) Video games as a tool to train visual skills. Restor Neurol Neurosci 26: 435-446.

4. Cole SW, Yoo DJ, Knutson B (2012) Interactivity and reward-related neural activation during a serious videogame. PLoS One 7: e33909.

5. Boas DA, Dale AM, Franceschini MA (2004) Diffuse optical imaging of brain activation: approaches to optimizing image sensitivity, resolution, and accuracy. Neuroimage 23 Suppl 1: 275-288.

6. Hock C, Muller-Spahn F, Schuh-Hofer F, et al. (1995) Age dependency of changes in cerebral hemoglobin oxygenation during brain activation: a near-infrared spectroscopy study. J Cereb Blood Flow Metab 15: 1103-1108

7. Hoshi Y, Tamura M (1993) Dynamic multichannel near-infrared optical imaging of human brain activity. J Appl Physiol 75: 1842-1846.

8. Hoshi Y, Onoe H, Watanabe Y, et al. (1994) Non-synchronous behavior of neuronal activity, oxidative metabolism and blood supply during mental tasks in man. Neurosci Lett 172: 129-133

9. Villringer A, Planck J, Hock C, et al (1993) Near infrared spectroscopy (NIRS): a new tool to study hemodynamic changes during activation of brain function in human adults. Neurosci Lett 154: 101-104

10. Villringer K, Minoshima S, Hock C, Obrig H, Ziegler S, et al. (1997) Assessment of local brain activation. A simultaneous PET and nearinfrared spectroscopy study. Adv Exp Med Biol 413: 149-153.

11. Herrmann MJ, Ehlis AC, Fallgatter AJ (2003) Frontal activation during a verbal-fluency task as measured by near-infrared spectroscopy. Brain Res Bull 61: 51-56.

12. Sato T, Ito M, Suto T, Kameyama M, Suda M, et al. (2007) Time courses of brain activation and their implications for function: a multichannel near-infrared spectroscopy study during finger tapping. Neurosci Res 58:297-304.

13. Shimada S, Hiraki K (2006) Infant's brain responses to live and televised action. Neuroimage 32: 930-939.

14. Nagamitsu S, Nagano M, Yamashita Y, Takashima S, Matsuishi T (2006) Prefrontal cerebral blood volume patterns while playing video games--a near-infrared spectroscopy study. Brain Dev 28: 315-21.

15. Nagamitsu S, Yamashita Y, Tanaka H, Matsuishi T (2012) Functional near-infrared spectroscopy studies in children. Biopsychosoc Med 6: 7.

16. Matsuda G, Hiraki K (2006) Sustained decrease in oxygenated hemoglobin during video games in the dorsal prefrontal cortex: a NIRS study of children. Neuroimage 29: 706-11.

17. Hoshi $Y$, Kobayashi N, Tamura M (2001) Interpretation of near-infrared spectroscopy signals: a study with a newly developed perfused rat brain model. J Appl Physiol 90: 1657-1662.

18. Strangman G, Culver JP, Thompson JH, Boas DA (2002) A quantitative comparison of simultaneous BOLD fMRI and NIRS recordings during functional brain activation. Neuroimage 17: 719-731.

19. Takeuchi M, Hori E, Takamoto K, Tran AH, Satoru K, et al. (2009) Brain Cortical Mapping by Simultaneous Recording of Functional Near Infrared Spectroscopy and Electroencephalograms from the Whole Brain During Right Median Nerve Stimulation. Brain Topogr 3:197-214.

20. Seiyama A, Hazeki O, Tamura M (1988) Noninvasive quantitative analysis of blood oxygenation in rat skeletal muscle. J Biochem 103: 419-424.

21. Fukui Y, Ajichi Y, Okada E (2003) Monte Carlo prediction of nearinfrared light propagation in realistic adult and neonatal head models. Appl Opt 42: 2881-2887.

22. Friston KJ, Frith CD, Frackowiak RS, Turner R (1995) Characterizing dynamic brain responses with fMRI: a multivariate approach. Neuroimage 2: 166-172.

23. Shimada S, Hiraki K, Oda I (2005) The parietal role in the sense of selfownership with temporal discrepancy between visual and proprioceptive feedbacks. Neuroimage 24: 1225-1232.

24. Takamoto K, Hori E, Urakawa S, Sakai S, Ichikawa A, et al. (2010) Cerebral hemodynamic responses induced by specific acupuncture sensations during needling at trigger points : a near - infrared spectroscopic study. Brain Topogr 3: 279-291

25. Schroeter ML, Bucheler MM, Muller K, UludaÄŸ K, Obrig H, et al. (2004) Towards a standard analysis for functional near-infrared imaging. Neuroimage 21: 283-290.

26. Suda M, Takei Y, Aoyama Y, Narita K, Sato T, et al. (2010) Frontopolar activation during face-to-face conversation: an in situ study using nearinfrared spectroscopy. Neuropsychologia 48: 441-7.

27. Sato T, Fukuda M, Kameyama M, Suda M, Uehara T, et al. (2012) Differential relationships between personality and brain function in monetary and goal-oriented subjective motivation: multichannel nearinfrared spectroscopy of healthy subjects. Psychiatry Clin Neurosci 66: 276-84.

28. Geschwind N (2010) Disconnexion syndromes in animals and man: Part I. 1965. Neuropsychol Rev 20: 128-157.

29. Ansari D (2008) Effects of development and enculturation on number representation in the brain. Nat Rev Neurosci 9: 278-291.

30. Newhart M, Trupe LA, Gomez Y, Cloutman L, Molitoris JJ, et al. (2012) Asyntactic comprehension, working memory, and acute ischemia in Broca's area versus angular gyrus. Cortex 48: 1288-1297.

31. Grabner RH, Reishofer G, Koschutnig K, Ebner F (2011) Brain correlates of mathematical competence in processing mathematical representations. Front Hum Neurosci 5: 130.

32. Price GR, Ansari D (2011) Symbol processing in the left angular gyrus: evidence from passive perception of digits. Neuroimage 57: 1205-1211.

33. Holloway ID, Price GR, Ansari D (2010) Common and segregated neural pathways for the processing of symbolic and nonsymbolic numerical magnitude: an fMRI study. Neuroimage 49: 1006-1017. 
Citation: Fujimori S, Terasawa K, Murata Y, Ogawa K, Tabuchi H, et al. (2015) Comparison of Cortical Activation during Mahjong Game Play in a Video Game Setting and a Real-life Setting. Biochem Anal Biochem 4: 164. doi:10.4172/2161-1009.1000164

Page 8 of 8

34. Chaim TM, Zhang T, Zanetti MV, da Silva MA, Louzã MR, et al. (2014) Multimodal magnetic resonance imaging study of treatment-naïve adults with attention-deficit/hyperactivity disorder. PLoS One 9: e110199.

35. He W, Liu D, Radua J, Li G, Han B, et al. (2015) Meta-analytic comparison between PIB-PET and FDG-PET results in Alzheimer's disease and MCI. Cell Biochem Biophys 71: 17-26.

36. Leménager T, Dieter J, Hill H, Koopmann A, Reinhard I, et al. (2014) Neurobiological correlat es of physical self-concept and selfidentification with avatars in addicted players of Massively Multiplayer Online Role-Playing Games (MMORPGs). Addict Behav 39: 1789-1797
37. Wan CL, Lin TK, Lu CH, Chang CS, Chen SD, et al. (2005) Mah-Jonginduced epilepsy: a special reflex epilepsy in Chinese society. Seizure 14: 19-22.

38. Kwan SY, Su MS (2000) Mah-jong epilepsy: a new reflex epilepsy. Zhonghua Yi Xue Za Zhi (Taipei) 63: 316-321.

39. Striano S, Coppola A, del Gaudio L, Striano P (2012) Reflex seizures and reflex epilepsies: old models for understanding mechanisms of epileptogenesis. Epilepsy Res 100: 1-11. 